\title{
Quantum non-Gaussianity and quantification of nonclassicality
}

\author{
B. Kühn* and W. Vogel \\ Arbeitsgruppe Quantenoptik, Institut für Physik, Universität Rostock, D-18051 Rostock, Germany
}

(Dated: March 23, 2022)

\begin{abstract}
The algebraic quantification of nonclassicality, which naturally arises from the quantum superposition principle, is related to properties of regular nonclassicality quasiprobabilities. The latter are obtained by non-Gaussian filtering of the Glauber-Sudarshan $P$ function. They yield lower bounds for the degree of nonclassicality. We also derive bounds for convex combinations of Gaussian states for certifying quantum non-Gaussianity directly from the experimentally accessible nonclassicality quasiprobabilities. Other quantum-state representations, such as $s$-parametrized quasiprobabilities, insufficiently indicate or even fail to directly uncover detailed information on the properties of quantum states. As an example, our approach is applied to multi-photon-added squeezed vacuum states.
\end{abstract}

\section{INTRODUCTION}

Uncovering structural information on quantum states is of fundamental importance for present quantum technologies. A prominent example for such characteristics is the classification of a state as classical or nonclassical [1]. Beyond this bivalent categorization, the amount and the kind of nonclassicality provided by various quantum resources is of great interest to figure out optimal experimental implementations of quantum technologies. In particular, a further state property - the quantum nonGaussianity [2-5] - is important for various applications in quantum technology [6-8].

In the past decades distinct measures for the quantification of nonclassicality have been proposed for harmonic oscillator quantum systems, such as light, the quantized motion of trapped atoms, and others. The early attempts rely on topological properties of quantum states; they are based on the distance of a state to the set of classical states. Such an approach can be based on different notions of the distance, such as the trace-norm-induced distance [9], the Monge distance [10], or the Hilbert-Schmidt distance [11]. The crucial point of such quantifications consists in its ambiguity. On the other hand, a quantification based on the number of superpositions of coherent states relies on the fundamental quantum superposition principle and yields an unambiguous quantification of nonclassicality [12]. Note that distance-based measures have also been studied for the quantification of non-Gaussianity [13-15].

Alternatively, the quantification problem has been addressed by using properties of quasiprobabilities [16, 17], which are full representations of the quantum states $[18,19]$. They may certify nonclassicality of various states [20, 21]. A powerful tool is the nonclassicality quasiprobability [22] - a regularized form of the GlauberSudarshan $P$ function $[23,24]$. It is designed such that it identifies any nonclassical state through negativities. This could be experimentally shown even for the strongly

* benjamin.kuehn2@uni-rostock.de singular squeezed states [25, 26]. In Ref. [27], the potential of a single-mode nonclassical state to generate quantum entanglement [28] by applying only linear optical elements was used to quantify nonclassicality. An algebraic approach for the quantification of nonclassicality was introduced, defining the degree of nonclassicality by the number of quantum superpositions of coherent states [29]. Based on the Schmidt number, an algebraic quantification was also used for quantum entanglement [28, 30, 31], which can be generalized to multipartite scenarios [32]. A unified quantification of nonclassicality and entanglement has been introduced [33], which directly relates the degree of nonclassicality to the Schmidt number of bipartite and even multipartite entangled states created by linear optical devices, such as an $N$ splitter. This is an important relation as entanglement is the basis for quantum technologies such as quantum information processing [34] and secure communication protocols [35]. For practical applications, the degree of nonclassicality can be verified both by a witness [36] and through the structure of the characteristic function of the Glauber-Sudarshan $P$ function [37]. However, these methods have not been developed for the purpose of uncovering quantum non-Gaussianity.

In the present paper we prove the usefulness of nonclassicality quasiprobabilities for certifying both quantum non-Gaussianity and the degree of nonclassicality. The single class of phase-space distributions under study uncovers both topological and algebraic quantifications of nonclassicality. We may uncover dissimilar features of arbitrary quantum states, such as nonclassicality, nonGaussianity, and the minimal number of quantum superpositions forming a given state. As the required phasespace functions are experimentally accessible, the present method also applies to state-of-the-art experiments.

\section{NONCLASSICALITY VERSUS QUANTUM NON-GAUSSIANITY}

The coherent states $|\alpha\rangle$ are well known to resemble the classical behavior of the harmonic oscillator and, hence, the classical character of light. Since mixing is a classical 
operation, a general state $\hat{\rho}$ is referred to as classical if it can be written as a mixture of coherent states,

$$
\hat{\rho}=\int d^{2} \alpha P_{\mathrm{cl}}(\alpha)|\alpha\rangle\langle\alpha|,
$$

with a classical probability distribution $P_{\mathrm{cl}}$. In other words, classical states are elements of the convex hull of the set of coherent states. In fact, any quantum state can be written in the coherent state basis [23, 24],

$$
\hat{\rho}=\int d^{2} \alpha P(\alpha)|\alpha\rangle\langle\alpha|
$$

However, the Glauber-Sudarshan function $P(\alpha)$ may fail to show the properties of a classical probability. Such states are referred to as nonclassical ones [1]. On this basis, any nonclassicality requires quantum superpositions of coherent states,

$$
\left|\psi_{r}\right\rangle=\sum_{j=1}^{r} \mu_{j}\left|\gamma_{j}\right\rangle
$$

where $\mu_{j}$ are nonzero complex numbers, $\left|\gamma_{j}\right\rangle$ are various coherent states, and $r$ is the number of superpositions. Now we may define the degree of nonclassicality as

$$
\kappa=r-1
$$

or as a monotonous function of this quantity [29]. This is an ambiguous quantification of nonclassicality, as an increase of nonclassicality is caused by a larger number of quantum superpositions. Pure states with nonclassicality degree $r-1$ can be written in the form (3) with the minimal number of $r$ superpositions of coherent states.

Extending these considerations, a general quantum state $\hat{\rho}$ has a nonclassicality degree $r-1$ if it is a classical mixture of pure states of a nonclassicality degree of at most $r-1$, i.e.,

$$
\hat{\rho}=\int d P_{\mathrm{cl}}\left(\left|\psi_{r}\right\rangle\right)\left|\psi_{r}\right\rangle\left\langle\psi_{r}\right| .
$$

In the case $r>1$, different such decompositions exist; thus, the nonclassicality degree refers to the minimal possible $r$. The states with a degree of nonclassicality of at most $r-1$ form a closed, convex set $\mathcal{M}_{r}$. This definition is straightforwardly generalized to multimode harmonic oscillator systems; see also Ref. [36].

A further important characteristics of quantum states is the non-Gaussianity. The Gaussian state $\left|G_{\boldsymbol{u}, \boldsymbol{\Sigma}}\right\rangle$ is fully determined through the first and second moment, namely, the mean value $\boldsymbol{u}$ and the covariance matrix $\boldsymbol{\Sigma}$. In principle, there are two types of non-Gaussianity. On the one hand, a non-Gaussian state can be obtained by properly mixing Gaussian states according to a classical probability distribution $P_{\mathrm{cl}}$, i.e.,

$$
\hat{\rho}=\int d P_{\mathrm{cl}}(\boldsymbol{u}, \boldsymbol{\Sigma})\left|G_{\boldsymbol{u}, \boldsymbol{\Sigma}}\right\rangle\left\langle G_{\boldsymbol{u}, \boldsymbol{\Sigma}}\right| .
$$

These states form also a closed, convex set $\mathcal{G}$ (see Ref. [5]). However, for quantum technologies this kind of non-Gaussianity is rather useless, since it originates from classical noise, such as phase randomization of Gaussian quantum states [38]. Of greater interest are quantum non-Gaussian states, $\hat{\rho} \notin \mathcal{G}$, whose non-Gaussianity is intrinsically quantum.

\section{STRUCTURAL INFORMATION IN PHASE-SPACE FUNCTIONS}

It is possible to use a witness approach to formulate criteria for the quantification of nonclassicality. A corresponding method was proposed [36], where the lower and upper bounds, $\bar{g}_{r}$ and $\underline{g}_{r}$, of the expectation value of a given Hermitian operator $\hat{L}$ are determined with respect to $\mathcal{M}_{r}$. If $\langle\hat{L}\rangle>\bar{g}_{r}$ or $\langle\hat{L}\rangle<\underline{g}_{r}$ for an unknown state, the nonclassicality degree of this state is shown to be necessarily greater than or equal to $r$. Equivalently, one introduces witness operators $\hat{W}_{r}=\bar{g}_{r} \hat{1}-\hat{L}$ and $\underline{\hat{W}}_{r}=\hat{L}-\underline{g}_{r} \hat{1}$, such that $\left\langle\hat{\bar{W}}_{r}\right\rangle \geq 0$ and $\left\langle\underline{\hat{W}}_{r}\right\rangle \geq 0$ for all states in $\overline{\mathcal{M}}_{r}$.

For the case $r=1$, a complete family of witness operators, $\{\hat{W}\}$, is already known [39], which is able to show that any state with nonclassicality degree $r-1>0$ (nonclassical state) cannot be written as a convex combination of pure nonclassicality degree-zero states (coherent states). Thus, these witnesses uncover all nonclassical effects of single- and multimode harmonic oscillator systems. In the single-mode case, they are of the form

$$
\hat{W}_{w, \alpha}=\hat{D}_{\alpha}^{\dagger} \hat{W}_{w} \hat{D}_{\alpha}
$$

with

$$
\hat{W}_{w}=\frac{1}{\pi^{2}} \int d^{2} \beta \Omega_{w}(\beta) e^{\beta \hat{a}^{\dagger}} e^{-\beta^{*} \hat{a}}
$$

and the coherent displacement operator $\hat{D}_{\alpha}=$ $\exp \left[\alpha \hat{a}^{\dagger}-\alpha^{*} \hat{a}\right]$. Here $\hat{a}$ and $\hat{a}^{\dagger}$ are the bosonic annihilation and creation operators, respectively. In total, there are only three free real parameters, a positive quantity $w$ and a complex number $\alpha$. The so-called nonclassicality filter $\Omega_{w}$ is chosen in such a way that the expectation value of $\hat{W}_{w, \alpha}$ exists for all states. Furthermore, its specific structure guarantees that this expectation value is non-negative for all classical states. A necessary and sufficient condition for a state being nonclassical is the existence of parameters $w$ and $\alpha$ such that $\left\langle\hat{W}_{w, \alpha}\right\rangle<0$.

The witnesses $\hat{W}_{w, \alpha}$ are associated with phase-space functions, the latter being an established method to visualize quantum effects through their negativities. In particular, the expectation value $P_{w}(\alpha)=\left\langle\hat{W}_{w, \alpha}\right\rangle$ can be regarded as a nonclassicality quasiprobability in phase space; it holds $\int d^{2} \alpha P_{w}(\alpha)=1$. For any positive value $w$ this function is nonsingular, as $\hat{W}_{w, \alpha}$ is a bounded 
TABLE I. For parameters $w$ in the range $\left[w_{\min }, w_{\max }\right]$ in each column, a lower bound for the degree of nonclassicality of up to $\kappa$ can be verified. In this range the overall supremum (infimum) of the nonclassicality quasiprobability $P_{w}(\alpha)$ is attained by a Fock state $|\bar{n}\rangle(|\underline{n}\rangle)$.

\begin{tabular}{c|ccccccc}
\hline \hline$w_{\min }$ & 1.200 & 1.550 & 1.795 & 2.027 & 2.239 & 2.436 & $\cdots$ \\
$w_{\max }$ & 1.550 & 1.795 & 2.027 & 2.239 & 2.436 & 2.619 & $\cdots$ \\
\hline $\bar{n}$ & 0 & 2 & 2 & 4 & 4 & 6 & $\cdots$ \\
$\underline{n}$ & 1 & 1 & 3 & 3 & 5 & 5 & $\cdots$ \\
$\kappa$ & 1 & 2 & 3 & 4 & 5 & 6 & $\cdots$ \\
\hline \hline
\end{tabular}

operator, and, thus, it is in principle accessible in experiments. In the limit $w \rightarrow \infty$ the function $P_{w}$ approaches the Glauber-Sudarshan $P$ function, which is our reference for nonclassicality. Note that $s$-parametrized quasiprobabilities [18], which correspond to a Gaussian filter $\Omega_{w}$ in Eq. (8), are only regular if the $s$ parameter is sufficiently small for the state under study. There is no $s$-parametrized quasiprobability which visualizes the nonclassicality of a squeezed vacuum state. Introducing non-Gaussian filters that decay more strongly than any Gaussian and that have a non-negative Fourier transform resolve this problem.

The witnesses $\hat{W}_{w, \alpha}$ in Eq. (7) together with Eq. (8) and, therefore, the associated nonclassicality quasiprobabilities provide a full test for nonclassicality. However, it is unknown yet to what extent structural state characteristics, such as the nonclassicality degree and quantum non-Gaussianity, can also be uncovered solely on the basis of these specific quantities. In order to approach this question, we combine the witness approach for quantifying nonclassicality on the basis of the quantum superposition principle [36] and the universal nonclassicality witness operators $\hat{W}_{w, \alpha}$, corresponding to regular phasespace functions. In the following we use the compact support filter defined in Refs. [39, 40], since the associated witness (8) has the closed form expression

$$
\hat{W}_{w}=: \frac{1}{\pi} \frac{\left[J_{1}\left(2 w \sqrt{\hat{a}^{\dagger} \hat{a}}\right)\right]^{2}}{\hat{a}^{\dagger} \hat{a}}:,
$$

where $J_{1}(\cdot)$ is the Bessel function of the first kind and : : : denotes normal ordering. This operator is diagonal in the Fock basis,

$$
\hat{W}_{w}=\sum_{n=0}^{\infty} c_{w, n}|n\rangle\langle n|,
$$

with the analytical coefficients [41]

$$
c_{w, n}=\frac{w^{2}}{\pi} \sum_{m=0}^{n} \frac{\left(-w^{2}\right)^{m}}{[(m+1) !]^{2}} \frac{n !}{(n-m) !}\left(\begin{array}{c}
2 m+2 \\
m
\end{array}\right) .
$$

Due to unitarity, the coherently displaced operator $\hat{W}_{w, \alpha}$ in Eq. (7) has the same eigenvalues as the operator $\hat{W}_{w}$. Therefore, the overall supremum and infimum of the nonclassicality quasiprobability $P_{w}(\alpha)$ for a fixed value of $w$ is attained for Fock states $|\bar{n}(w)\rangle$ and $|\underline{n}(w)\rangle$, respectively (see Appendix A). Table I shows that the supremum is attained for even numbers $\bar{n}$, while the infimum is attained for odd numbers $\underline{n}$. These numbers increase sequently and change alternately whenever the parameter $w$ exceeds a critical value. Fock states $|n\rangle$ have the nonclassicality degree $\kappa=n$, as they are representable as a quantum superposition of $n+1$ coherent states [42],

$$
|n\rangle=\lim _{\epsilon \rightarrow 0} \mathcal{C}_{n}(\epsilon) \sum_{k=0}^{n} e^{-2 \pi i k n /(n+1)}\left|\epsilon e^{2 \pi i k /(n+1)}\right\rangle,
$$

weighted equally and their amplitudes located on a circle in the complex plane with radius tending to zero; $\mathcal{C}_{n}$ provides the correct normalization. This is a maximally nonclassical state in the topological picture. Consequently, for given $w$ a nonclassicality degree up to $\max [\bar{n}(w), \underline{n}(w)]$ can be certified by means of the nonclassicality quasiprobability $P_{w}(\alpha)$.

Let us compare this result with the case of $s$ parametrized quasiprobabilities. In the parameter range $s \leq 0$, where these functions are always regular, the overall supremum is attained by the vacuum state and the overall infimum by the single-photon state. In fact, these quasiprobabilities uncover only the presence of some nonclassicality for $s>-1$ without resolving a nonclassicality degree. This clearly demonstrates, that the $s$ parametrized quasiprobabilities not only provide an incomplete nonclassicality test; they also do not yield more specific structural insight.

Now, we determine the upper and lower bounds, $\bar{g}_{r}$ and $g_{r}$, of the nonclassicality quasiprobability $P_{w}(\alpha)$ associated with the witness operator (9) over the set $\mathcal{M}_{r}$. Mixing cannot shift the optimum, thus, the optimization can run over pure states $\left|\psi_{r}\right\rangle$ defined in Eq. (3). Furthermore, the coherent displacement in Eq. (7), can be included in the states. In total, the quantity to be optimized reads as

$\left\langle\psi_{r}\left|\hat{W}_{w}\right| \psi_{r}\right\rangle=\frac{1}{\pi} \sum_{\ell=1}^{r} \sum_{j=1}^{r} \mu_{\ell}^{*} \mu_{j}\left[J_{1}\left(2 w \sqrt{\gamma_{\ell}^{*} \gamma_{j}}\right)\right]^{2} \frac{\left\langle\gamma_{\ell} \mid \gamma_{j}\right\rangle}{\gamma_{\ell}^{*} \gamma_{j}}$.

The complex coherent amplitudes $\left(\gamma_{1}, \ldots, \gamma_{r}\right)^{T}$ and complex coefficients $\left(\mu_{1}, \ldots, \mu_{r}\right)^{T}$ are the optimization parameters, which have to fulfill the normalization constraint $\left\langle\psi_{r} \mid \psi_{r}\right\rangle=1$. We perform our analysis up to $r=6$, requiring to optimize in total up to 22 free real parameters, which is done by a genetic search [43] together with a gradient descent [44]. In a similar way, the upper and lower bounds, $\bar{g}_{\mathrm{G}}$ and $g_{\mathrm{G}}$, of $P_{w}(\alpha)$ with respect to the Gaussian convex hull $\mathcal{G}$ are obtained by an optimization over pure Gaussian states. The latter are squeezed coherent states, defined by the complex squeezing parameter and complex coherent displacement amplitude.

The result is shown in Fig. 1 and gives a rich amount of insight. For better visualization of the relative effects, 


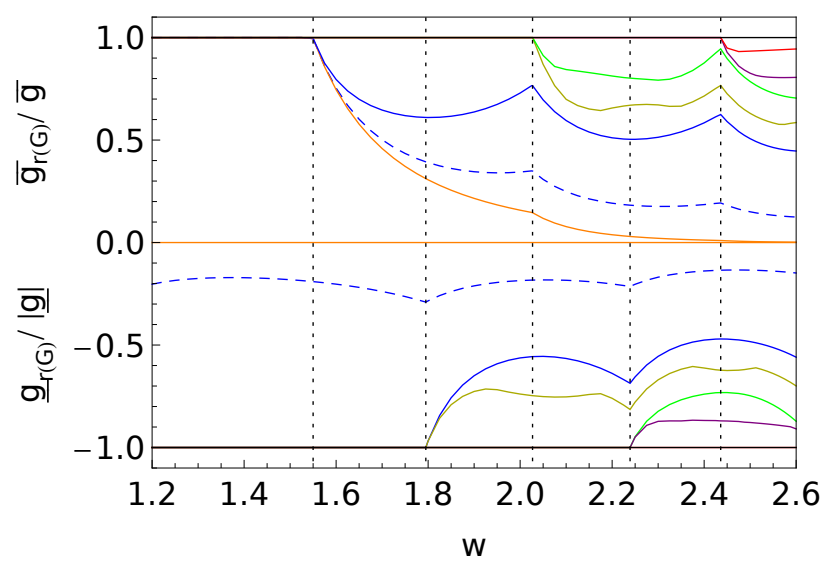

FIG. 1. (Color online) The maximal (minimal) values $\bar{g}_{r}\left(\underline{g}_{r}\right)$ for $r=1, \ldots, 6$ as a function of the parameter $w$. Solid lines with positive (negative) function values from bottom to top (from top to bottom): $r=1$ (orange), $r=2$ (blue), $r=3$ (dark yellow), $r=4$ (green), $r=5$ (purple), and $r=6$ (red). We normalized the bounds to the overall supremum $\bar{g}$ (infimum $g$ ). The maximal (minimal) values $\bar{g}_{\mathrm{G}}\left(\underline{g}_{\mathrm{G}}\right)$ attained by any mixture of Gaussian states for a given $w$ are illustrated by the dashed blue lines, normalized in the same way. The vertical dotted lines mark the critical values of $w$, where a next higher degree of nonclassicality $\kappa$ can be certified.

$\bar{g}_{r}(w)$ is normalized by the overall supremum, and $\underline{g}_{r}(w)$ by the modulus of the overall infimum of the nonclassicality quasiprobability. One observes a splitting of the supremum (infimum) into three separated levels (trifurcation) whenever the increasing parameter $w$ traverses critical values, which turn out to be identical to the values where the overall supremum (infimum) is attained by another Fock state (see Table I). This systematics most likely persists also for $w>2.6$, which allows us to arbitrarily increase the resolvable nonclassicality degree. The parameter $w$ should be chosen as large as necessary but as small as possible, as experimental data sampling noise increases with increasing $w$.

Interestingly, in the case of $r=2$, the bounds $\bar{g}_{r}$ and $\underline{g}_{r}$ are attained by even and odd coherent states, $\left|\gamma_{ \pm}\right\rangle=$ $\overline{\mathcal{N}}_{ \pm}(|\gamma\rangle \pm|-\gamma\rangle)$, respectively, which are identified to be maximally nonclassical in the sense of (distance-based) topological nonclassicality measures. The upper and lower bounds, $\bar{g}_{\mathrm{G}}$ and $\underline{g}_{\mathrm{G}}$, for the set $\mathcal{G}$ of mixtures of Gaussian states are also contained in Fig. 1 and they are well separated from the levels $\bar{g}_{r}\left(g_{r}\right)$; for further details on the optimal states see Appendix B. Since $\mathcal{G}$ contains also the nonclassical squeezed states, it holds $g_{\mathrm{G}}<g_{1}=0$, and $\bar{g}_{\mathrm{G}}$ also exceeds the classical level $\overline{\bar{g}}_{1}$. Note that, even for arbitrarily weak squeezing, these states are quantum superpositions of an infinite number of coherent states, referring to them as maximally nonclassical from the perspective of algebraic nonclassicality measures. Our results clearly show, that the nonclassicality quasiprobabilities uncover both the degree of nonclassicality and quantum non-Gaussianity without additional means.

\section{PHOTON-ADDED SQUEEZED STATES}

The structure of the operator $\hat{W}_{w, \alpha}$ in Eq. (7) allows us to certify especially Fock-like states - states having a high overlap with a Fock state - to exceed the nonclassicality degree bounds $\bar{g}_{r}$ and $\underline{g}_{r}$. As an example, we consider the non-Gaussian states obtained by multiphoton additions

(a)

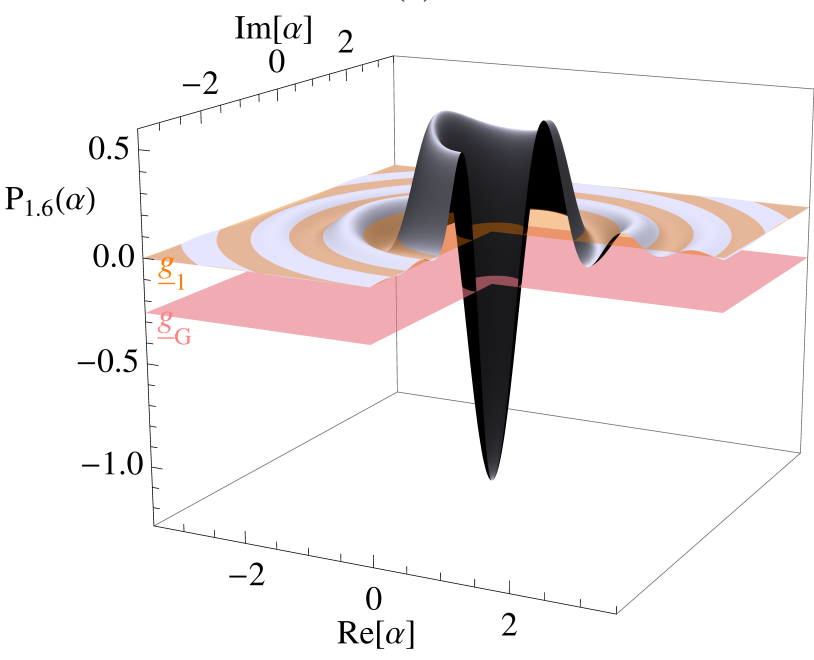

(b)

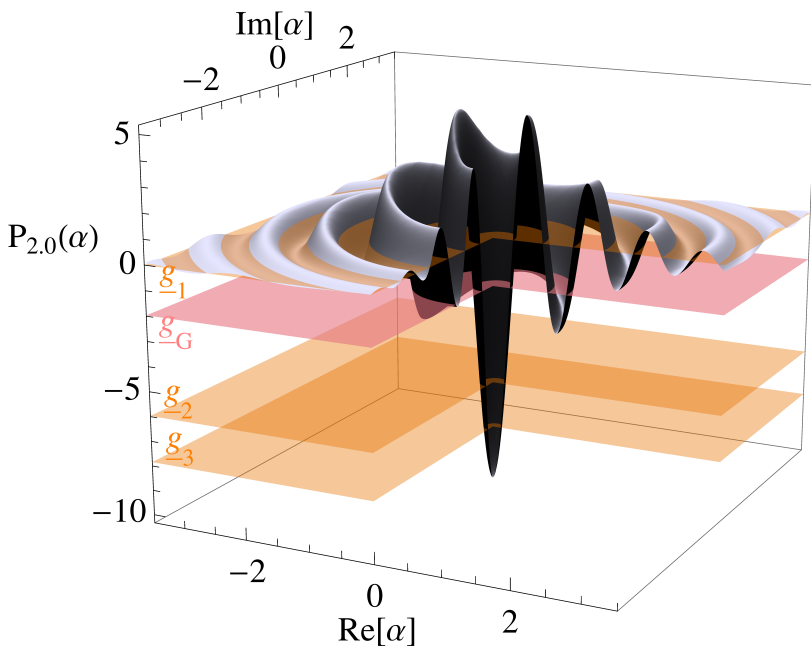

FIG. 2. (Color online) Nonclassicality quasiprobability $P_{w}(\alpha)$ of an $m$-photon-added squeezed vacuum state for odd $m$ and squeezing parameter $\xi=0.1$ : (a) $m=1, w=1.6$; (b) $m=3, w=2.0$. The lower bounds $\underline{g}_{r}$ for states with a nonclassicality degree of at most $r-1$ are given by the transparent orange planes. The lower bound for convex combinations of Gaussian states, $\underline{g}_{\mathrm{G}}$, is indicated by the transparent pink plane. For better visualization, we cut out the front-right quadrant. 
to an initial Gaussian squeezed state, i.e.,

$$
|m, \xi\rangle=\mathcal{N}_{m, \xi} \hat{a}^{\dagger m} \hat{S}(\xi)|0\rangle,
$$

with the unitary squeezing operator $\hat{S}(\xi)=$ $\exp \left[\frac{1}{2}\left(\xi^{*} \hat{a}^{2}-\xi \hat{a}^{\dagger 2}\right)\right]$. The squeezing parameter is chosen to be $\xi=0.1$ and $m$ is the number of added photons. The prefactor $\mathcal{N}_{m, \xi}$ ensures the correct normalization.

(a)

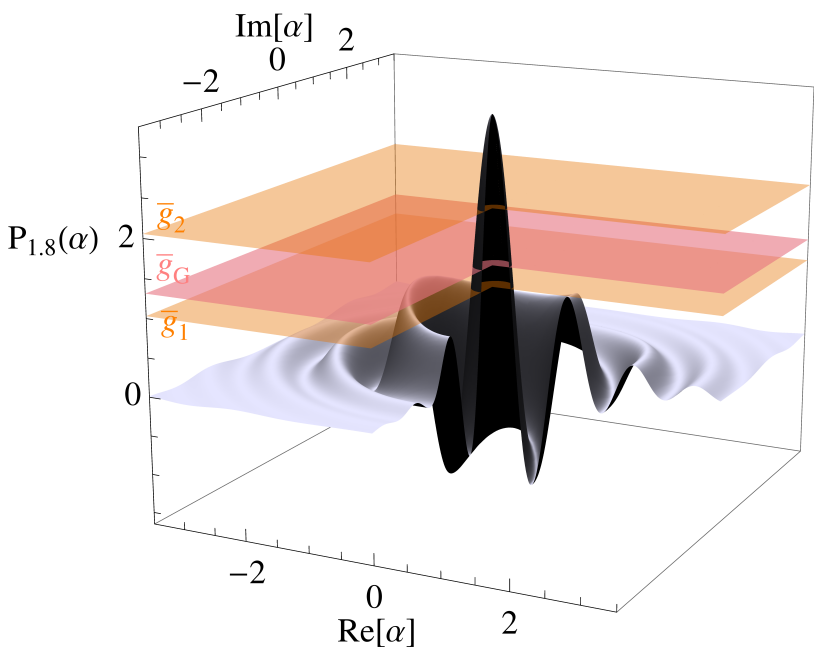

(b)

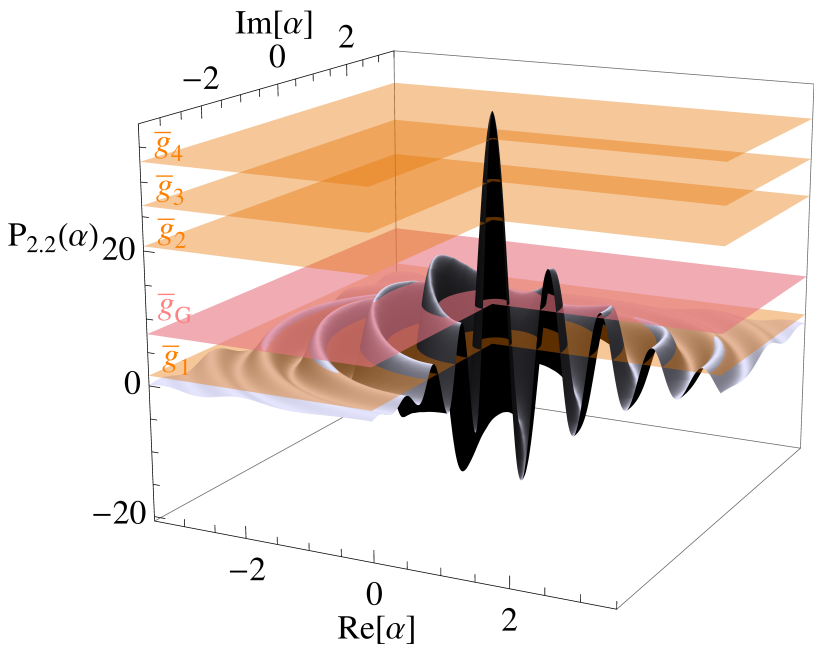

FIG. 3. (Color online) Nonclassicality quasiprobability of an $m$-photon-added squeezed vacuum state for even $m$ and squeezing parameter $\xi=0.1$ : (a) $m=2, w=1.8$; (b) $m=4$, $w=2.2$. The upper bounds $\bar{g}_{r}$ for states with a nonclassicality degree of at most $r-1$ are given by the transparent orange planes. The upper bound for convex combinations of Gaussian states, $\bar{g}_{\mathrm{G}}$, is indicated by the transparent pink plane. For better visualization, we cut out the front-right quadrant.

Figures 2(a) and 2(b) show the nonclassicality quasiprobability of a single- and a three-photon-added squeezed vacuum state, respectively. For the singlephoton addition, the phase-space function falls below $\underline{g}_{1}=0$ but not below $\underline{g}_{2}$. Thus, nonclassicality is certified $(\kappa>0)$, but no information on the nonclassicality degree is extracted. One clearly observes that through the addition of three photons the quasiprobability falls below the lower bound $g_{3}$. Accordingly, this state has at least the nonclassicality degree $\kappa=3$; i.e., it is composed of a quantum superposition of at least four coherent states. Both states are certified to be quantum non-Gaussian ones, since $P_{w}(\alpha)$ penetrates $\underline{g}_{\mathrm{G}}$.

In addition, we present in Figs. 3(a) and 3(b) the results for photon-added squeezed vacuum states with two and four added photons and the same squeezing parameter as in Fig. 2. Since $P_{w}(\alpha)$ in Fig. 3(a) exceeds the upper bound $\bar{g}_{2}$, the two-photon-added squeezed vacuum state is shown to have a nonclassicality degree of at least 2. The nonclassicality quasiprobability in Fig. 3 (b) exceeds the upper bound $\bar{g}_{4}$ and, accordingly, the four-photon-added squeezed vacuum state has a nonclassicality degree of at least 4 . Both states strongly exceed the range allowed for convex combinations of Gaussian states; they are therefore shown to be quantum nonGaussian.

\section{CONCLUSIONS}

In conclusion, the nonclassicality quasiprobabilities were so far known to fully verify the nonclassicality of quantum states. Beyond this, we have shown that they additionally uncover important structural quantum characteristics of the state. Beneficially, both the degree of nonclassicality and the quantum non-Gaussianity are accessible via this single quantity. The nonclassicality degree, detected by our method, is useful for applications in quantum technologies, as the nonclassicality degree of a single-mode beam forwarded to the input of an $N$ splitter coincides with the amount of multipartite entanglement produced in the outputs. Most importantly, the structure of nonclassicality quasiprobabilities uncovers both algebraic and topological aspects of nonclassicality.

\section{ACKNOWLEDGMENTS}

The authors are grateful to S. Ryl and J. Sperling for enlightening discussions. This work has been supported by the European Commission through the project QCUMbER (Quantum Controlled Ultrafast Multimode Entanglement and Measurement), Grant No. 665148. 


\section{APPENDIX}

\section{Appendix A: Overall boundaries of nonclassicality quasiprobabilities}

In this section the overall maximization and minimization of the nonclassicality quasiprobability $P_{w}(\alpha)$ with respect to all quantum states is studied. For reasons described in the main text, the overall supremum $\bar{g}(w)$ and infimum $g(w)$ of the nonclassicality quasiprobability $P_{w}(\alpha)$ for a fixed value of $w$ is attained for Fock states $|\bar{n}(w)\rangle$ and $|\underline{n}(w)\rangle$, respectively, with

$$
\begin{aligned}
& \bar{n}(w)=\arg \max _{n}\left[c_{w, n}\right], \\
& \underline{n}(w)=\arg \min _{n}\left[c_{w, n}\right], \\
& \bar{g}(w)=\max _{n}\left[c_{w, n}\right] \\
& \underline{g}(w)=\min _{n}\left[c_{w, n}\right]
\end{aligned}
$$

where the coefficients $c_{w, n}$ are defined in Eq. (11) of the main text.

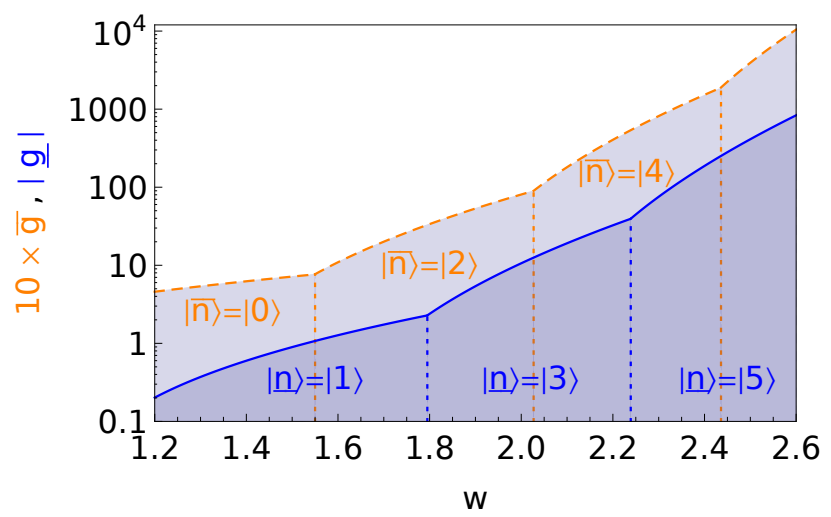

FIG. 4. (Color online) The supremum $\bar{g}$ (dashed orange line) and the modulus of the infimum $g$ (solid blue line) of $P_{w}$, which are attained by Fock states $|\bar{n}\rangle$ and $|\underline{n}\rangle$ as a function of the parameter $w$. Note that the supremum is depicted with a factor of 10 for better visual separation of both curves.

These quantities are illustrated in Fig. 4 on a logarithmic scale. Interestingly, one observes a well-ordered structure. The supremum is attained for Fock states with even number, while the infimum is attained for odd ones. The change of $\bar{n}(\underline{n})$ at the critical values accompanies a discontinuity of the derivative of $\bar{g}(w)(g(w))$ with respect to $w$.

\section{Appendix B: Optimal states}

It turns out that even (odd) coherent states,

$$
\left|\gamma_{ \pm}\right\rangle=\frac{1}{\sqrt{2\left(1 \pm e^{-2|\gamma|^{2}}\right)}}(|\gamma\rangle \pm|-\gamma\rangle)
$$

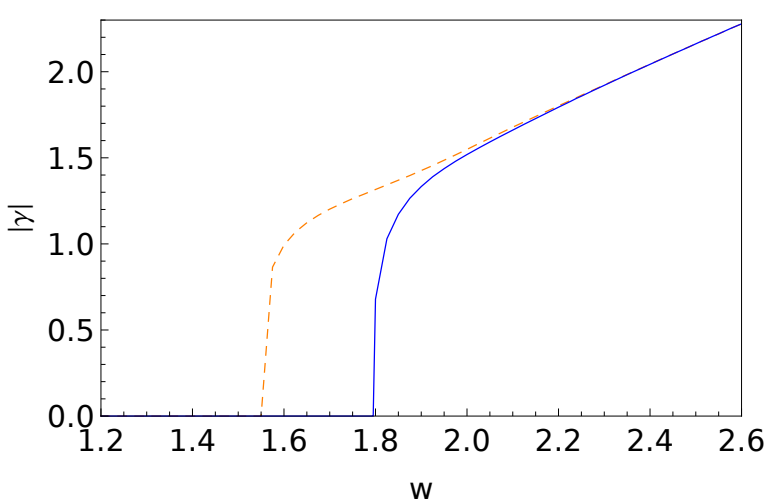

FIG. 5. (Color online) Dashed orange line (solid blue line): Amplitude $|\gamma|$ of the even (odd) coherent state, which maximizes (minimizes) the nonclassicality quasiprobability $P_{w}(\alpha)$ with respect to the set $\mathcal{M}_{2}$ of states with a nonclassicality degree of $\kappa=1$ as a function of the parameter $w$.

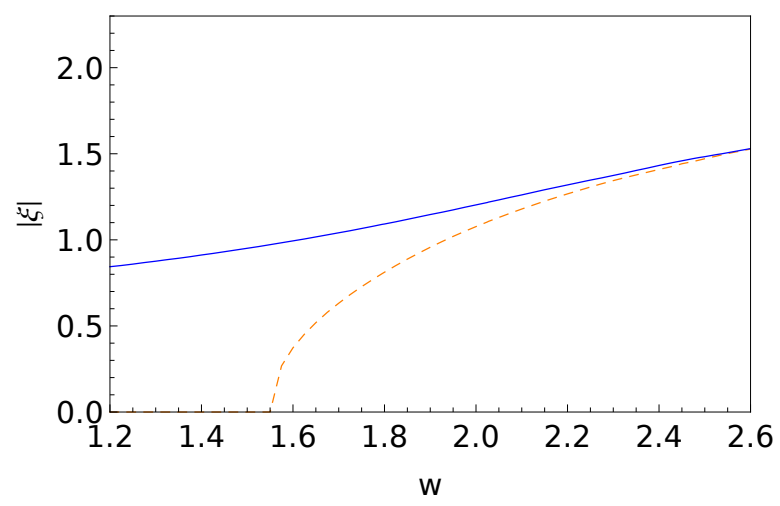

FIG. 6. (Color online) Dashed orange line (solid blue line): Absolute value of the squeezing parameter $\xi$ of the squeezed vacuum state, which maximizes (minimizes) the nonclassicality quasiprobability $P_{w}(\alpha)$ with respect to the set $\mathcal{G}$ of mixtures of Gaussian states as a function of the parameter $w$.

where the "+" corresponds to even ones and the "-" to odd ones, maximize (minimize) the nonclassicality quasiprobability $P_{w}(\alpha)$ with respect to the set $\mathcal{M}_{2}$ of states with nonclassicality degree $\kappa=1$. The absolute value of the amplitude $\gamma$, for which the maximum and minimum are obtained, is shown in Fig. 5 as a function of $w$. The two curves approach each other for increasing parameter $w$. Accordingly, for large $w$ the maximum and minimum are attained for even and odd coherent states with nearly the same amplitude $\gamma$. This optimal amplitude increases with increasing $w$. For $w<1.55$ the optimal amplitude of the even coherent state, corresponding to the maximum of $P_{w}(\alpha)$, is $\gamma=0$ in Eq. (B1), which is the vacuum state. For $w<1.795$ the amplitude of the odd coherent state providing the minimum is $\gamma=0$, which according to Eq. (B1) coincides with the single-photon state.

The nonclassicality quasiprobability is optimized with respect to the set $\mathcal{G}$ of mixtures of Gaussian states for 
squeezed vacuum states [Eq. (14) with $m=0$ in the main text]. The absolute value of the squeezing parameter $\xi$, which yields the maximum and minimum of $P_{w}(\alpha)$, is illustrated in Fig. 6 as a function of $w$. In the analyzed range of $w$, the minimum of the nonclassicality quasiprobability is attained for larger $|\xi|$ than the maxi- mum. Furthermore, for $w<1.55$ one obtains the maximum for the vacuum state, which corresponds to $\xi=0$. We also observe that the maximum and minimum are obtained for the same increasing squeezing parameter in the limit of a large parameter $w$.
[1] U. M. Titulaer and R. J. Glauber, Correlation functions for coherent fields, Phys. Rev. 140, B676 (1965).

[2] M. Barbieri, N. Spagnolo, M. G. Genoni, F. Ferreyrol, R. Blandino, M. G. A. Paris, P. Grangier, and R. Tualle-Brouri, Non-Gaussianity of quantum states: An experimental test on single-photon-added coherent states, Phys. Rev. A 82, 063833 (2010).

[3] M. Ježek, I. Straka, M. Mičuda, M. Dušek, J. Fiurášek, and R. Filip, Experimental Test of the Quantum NonGaussian Character of a Heralded Single-Photon State, Phys. Rev. Lett. 107, 213602 (2011).

[4] R. Filip and L. Mišta, Jr., Detecting Quantum States with a Positive Wigner Function beyond Mixtures of Gaussian States, Phys. Rev. Lett. 106, 200401 (2011).

[5] M. G. Genoni, M. L. Palma, T. Tufarelli, S. Olivares, M. S. Kim, and M. G. A. Paris, Detecting quantum nonGaussianity via the Wigner function, Phys. Rev. A 87, 062104 (2013).

[6] I. Straka, A. Predojević, T. Huber, L. Lachman, L. Butschek, M. Miková, M. Mičuda, G. S. Solomon, G. Weihs, M. Ježek, and R. Filip, Quantum non-Gaussian Depth of Single-Photon States, Phys. Rev. Lett. 113, 223603 (2014).

[7] M. Lasota, R. Filip, and V. Usenko, Sufficiency of quantum non-Gaussianity for discrete-variable quantum key distribution over noisy channels, Phys. Rev. A 96, 012301 (2017).

[8] I. Straka, L. Lachman, J. Hloušek, M. Miková, M. Mičuda, M. Ježek, and R. Filip, Quantum non-Gaussian multiphoton light, npj Quantum Inf. 4, 4 (2018).

[9] M. Hillery, Nonclassical distance in quantum optics, Phys. Rev. A 35, 725 (1987).

[10] K. Życzkowski and W. Słomczyński, The Monge distance between quantum states, J. Phys. A: Math. Gen. 31, 9095 (1998).

[11] V. V. Dodonov, O. V. Man'ko, V. I. Man'ko, and A. Wünsche, Hilbert-Schmidt distance and non-classicality of states in quantum optics, Journal of Modern Optics 47, 633 (2009)

[12] J. Sperling and W. Vogel, Convex ordering and quantification of quantumness, Phys. Scr. 90, 074024 (2015).

[13] M. G. Genoni, M. G. A. Paris, K. Banaszek, Measure of the non-Gaussian character of a quantum state, Phys. Rev. A 76, 042327 (2007).

[14] M. G. Genoni, M. G. A. Paris, K. Banaszek, Quantifying the non-Gaussian character of a quantum state by quantum relative entropy, Phys. Rev. A 78, 060303(R) (2008).

[15] J. Park, J. Lee, S.-W. Ji, and H. Nha, Quantifying nonGaussianity of quantum-state correlation, Phys. Rev. A 96, 052324 (2017).

[16] C. T. Lee, Measure of the nonclassicality of nonclassical states, Phys. Rev. A 44, R2775(R) (1991).
[17] N. Lütkenhaus and S. M. Barnett, Nonclassical effects in phase space, Phys. Rev. A 51, 3340 (1995).

[18] K. E. Cahill and R. J. Glauber, Ordered Expansions in Boson Amplitude Operators, Phys. Rev. 177, 1857 (1969).

[19] G. S. Agarwal and E. Wolf, Calculus for Functions of Noncommuting Operators and General Phase-Space Methods in Quantum Mechanics. II. Quantum Mechanics in Phase Space, Phys. Rev. D 2, 2187 (1970).

[20] D. Leibfried, D. M. Meekhof, B. E. King, C. Monroe, W. M. Itano, and D. J. Wineland, Experimental Determination of the Motional Quantum State of a Trapped Atom, Phys. Rev. Lett. 77, 4281 (1996).

[21] S. Deléglise, I. Dotsenko, C. Sayrin, J. Bernu, M. Brune, J.-M. Raimond, and S. Haroche, Reconstruction of nonclassical cavity field states with snapshots of their decoherence, Nature 455, 510 (2008).

[22] T. Kiesel and W. Vogel, Nonclassicality filters and quasiprobabilities, Phys. Rev. A 82, 032107 (2010).

[23] R. J. Glauber, Coherent and incoherent states of the radiation field, Phys. Rev. 131, 2766 (1963).

[24] E. C. G. Sudarshan, Equivalence of Semiclassical and Quantum Mechanical Descriptions of Statistical Light Beams, Phys. Rev. Lett. 10, 277 (1963).

[25] T. Kiesel, W. Vogel, B. Hage, and R. Schnabel, Direct sampling of negative quasiprobabilities of a squeezed state, Phys. Rev. Lett. 107, 113604 (2011).

[26] E. Agudelo, J. Sperling, W. Vogel, S. Köhnke, M. Mraz, and B. Hage, Continuous sampling of the squeezed-state nonclassicality, Phys. Rev. A 92, 033837 (2015).

[27] J. K. Asbóth, J. Calsamiglia, and H. Ritsch, Computable Measure of Nonclassicality for Light, Phys. Rev. Lett. 94, $173602(2005)$

[28] R. Horodecki, P. Horodecki, M. Horodecki, and K. Horodecki, Quantum entanglement, Rev. Mod. Phys. 81, 865 (2009).

[29] C. Gehrke, J. Sperling, and W. Vogel, Quantification of nonclassicality, Phys. Rev. A 86, 052118 (2012).

[30] B. M. Terhal and P. Horodecki, Schmidt number for density matrices, Phys. Rev. A 61, 040301(R) (2000).

[31] A. Sanpera, D. Bruß, and M. Lewenstein, Schmidtnumber witnesses and bound entanglement, Phys. Rev. A 63, 050301(R) (2001).

[32] J. Eisert and H. J. Briegel, Schmidt measure as a tool for quantifying multiparticle entanglement, Phys. Rev. A 64, 022306 (2001).

[33] W. Vogel and J. Sperling, Unified quantification of nonclassicality and entanglement, Phys. Rev. A 89, 052302 (2014).

[34] M. A. Nielsen and I. L. Chuang, Quantum Computation and Quantum Information (Cambridge University Press, Cambridge, England, 2000).

[35] N. Gisin and R. Thew, Quantum communication, Nat. 
Photon. 1, 165 (2007).

[36] M. Mraz, J. Sperling, W. Vogel, and B. Hage, Witnessing the degree of nonclassicality of light, Phys. Rev. A 90, 033812 (2014)

[37] S. Ryl, J. Sperling, and W. Vogel, Quantifying nonclassicality by characteristic functions, Phys. Rev. A 95, 053825 (2017).

[38] A. Franzen, B. Hage, J. DiGuglielmo, J. Fiurášek, and R. Schnabel, Experimental Demonstration of Continuous Variable Purification of Sqeezed States, Phys. Rev. Lett. 97, 150505 (2006).

[39] T. Kiesel and W. Vogel, Universal nonclassicality witnesses for harmonic oscillators, Phys. Rev. A 85, 062106 (2012).

[40] B. Kühn and W. Vogel, Visualizing nonclassical effects in phase space, Phys. Rev. A 90, 033821 (2014).
[41] T. Kiesel and W. Vogel, Complete nonclassicality test with a photon-number-resolving detector, Phys. Rev. A 86, 032119 (2012).

[42] J. Janszky, P. Domokos, and P. Adam, Coherent states on a circle and quantum interference, Phys. Rev. A 48, 2213 (1993).

[43] R. L. Haupt and S. E. Haupt, Practical Genetic Algorithms, 2nd Ed. (John Wiley \& Sons, Hoboken, NJ, 2004).

[44] J. A. Snyman, Practical Mathematical Optimization: An Introduction to Basic Optimization Theory and Classical and New Gradient-Based Algorithms, 2nd Ed. (Springer Science \& Business Media, United States of America, 2005). 